\title{
Modelo empírico para previsión de la temperatura superficial del mar peruano
}

\section{Empirical model for forecast the Peruvian sea surface temperature}

\author{
Benjamin Matellini ${ }^{1}$, Jorge Tam ${ }^{1}$ y Carlos Quispe ${ }^{1}$
}

\begin{abstract}
${ }^{1}$ Centro de Investigaciones en Modelado Oceanográfico y Biológico Pesquero (CIMOBP), Instituto del Mar del Perú (IMARPE), Apdo. 22, Callao, Perú.

Email Jorge Tam: jtam@imarpe.gob.pe
\end{abstract}

Presentado: $\quad 11 / 12 / 2005$ Aceptado: $\quad 25 / 02 / 2007$

\section{Resumen}

En el presente trabajo se estudia un modelo empírico basado en el volumen de agua cálida en el Pacífico ecuatorial que se aplica para hacer previsiones de temperatura superficial del mar frente a Perú, lo cual sirve como una herramienta de alerta temprana de los efectos de El Niño. La relación del volumen de agua cálida con la profundidad de la isoterma de $20^{\circ} \mathrm{C}$ es mayor en el Pacífico central en la latitud cero. Los cambios de este volumen afectan después varios meses la temperatura superficial del mar frente a Perú, particularmente cuando este volumen presenta anomalías positivas. El modelo logra estimar el momento en que se inicia el aumento de temperatura superficial del mar asociado a los efectos de El Niño en la Región Niño 1 costera, el momento de ocurrencia del pico de los eventos, y las tendencias a mediano plazo.

Palabras claves: Modelo empírico, volumen de agua cálida, Pacífico Ecuatorial, temperatura superficial, El Niño.

\section{Abstract}

In the present work an empirical model based on the warm water volume in the Equatorial Pacific Ocean is studied as a tool to forecast variations of sea surface temperature off the coast of Perú, which will be useful as a tool for giving early warnings of El Niño effects. The relation between the warm water volume and the depth of the $20^{\circ} \mathrm{C}$ isotherm is greater in the Central Pacific along latitude $0^{\circ}$. Several months later the changes of this volume affect the sea surface temperature off Perú particularly when its anomalies are positive. The model estimates the time when sea surface temperature begins to rise associated with El Niño effects in the coastal Niño 1 Region, the time when the event peaks, and the medium term trends.

Keywords: Empirical model, warm water volume, Equatorial Pacific Ocean, surface temperature, El Niño

\section{Introducción}

El Niño-Oscilación del Sur (ENOS) es un evento océanoatmosférico de macro-escala, recurrente en una escala de tiempo interanual, que produce impactos en varias regiones del mundo, en particular la región oriental del Pacífico ecuatorial la cual es más la afectada. Numerosos trabajos de investigación se han realizado con el fin de poder predecir el evento no sólo por el interés científico sino también por su enorme implicancia socioeconómica (Arntz y Fahrbach 1996).

Una de las primeras hipótesis sobre ENOS fue la postulada por Bjerknes (1969) quien sostuvo que el evento es el resultado de interacciones océano-atmosféricas en el Pacífico tropical y que los vientos alisios son producto del gradiente térmico Oeste-Este del océano. Estos vientos, por su parte, contribuyen a fortalecer este gradiente térmico, produciendo un mayor enfriamiento de las masas de aguas del Pacífico Este. Otras hipótesis bastante aceptadas (Cane y Zebiak, 1985 y Wyrtki, 1986) propusieron que hay una recarga de agua cálida antes de un El Niño y que después del evento el contenido de calor es bajo y esto equivale a La Niña. La recarga de agua cálida se puede considerar como la expansión de la piscina caliente del Oeste. Esta expansión está confinada al Pacífico Oeste y se observa principalmente en el espesor (vertical) de la región caliente así como en el sentido meridional (McPhaden 2003). La expansión zonal es mínima debido a que la piscina caliente del Oeste tiene la masa continental como límite en el Oeste, mientras que por el Este es contenida por los vientos alisios, los cuales incluso se intensifican en el sentido zonal hacia el Oeste debido a un aumento en la gradiente térmica zonal. En el caso de intensifi- carse los vientos alisios, la extensión zonal de la piscina caliente disminuiría. Al ocurrir El Niño, esta piscina caliente o agua cálida se desplaza hacia el Pacífico Este. Dado que existe una demora en el traslado de agua cálida del Oeste hacia el Este, es posible que el análisis de su movimiento sirva como herramienta para la previsión de la temperatura en el Pacífico Este, y en especial frente a Perú. A pesar de que esta relación pueda estar afectada por otros procesos físicos, es necesario explorar su efectividad como herramienta de previsión.

Los cambios espaciales de esta agua cálida se hacen evidentes por su avance hacia el Este lo cual resulta en la profundización de la termoclina conforme se expande hacia esa dirección, por lo que es importante identificar el punto zonal donde el cambio de la profundidad de la termoclina está mas relacionado al inicio de un El Niño. Al realizar este análisis se debe considerar que los flujos meridionales que convergen o divergen en el ecuador también influyen en la profundidad de la termoclina. Es posible que estos flujos en algún momento puedan configurar el océano ecuatorial de tal manera que el sistema océano - atmósfera responda con un El Niño ante pulsos de vientos del Oeste.

El desplazamiento de agua cálida del Oeste hacia el Este asociado a El Niño, se puede iniciar hipotéticamente por ondas Kelvin dependiendo del estado del océano en el momento que se producen. Estas ondas pueden ocurrir por pulsos de vientos hacia el Este en el Pacífico Oeste asociados a la oscilación Madden-Julian, o por el reflejo de una onda Rossby a la altura de la línea ecuatorial. El Pacífico Oeste y Central es muy sensible a cambios de temperatura y un ligero calentamiento debilita (fortalece) de inmediato el componente zonal de los vientos que 


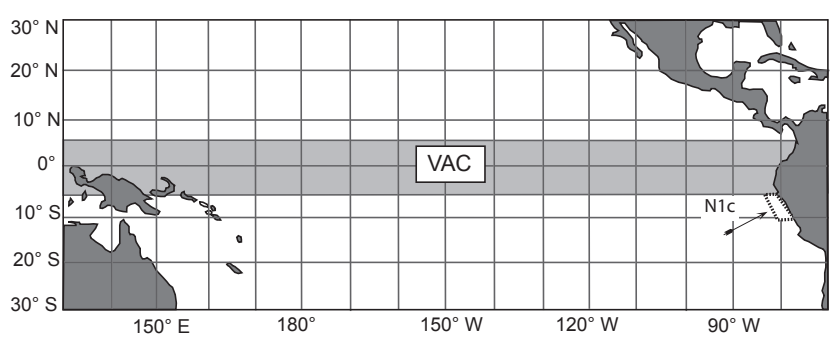

Figura 1. Zonas de estudio en el Océano Pacífico. El rectángulo gris representa el espacio dentro del cual se define el volumen de agua calida (VAC). El rectángulo pequeño frente a Perú es la Región Niño 1 costera (N1c).

soplan hacia el Oeste (Este). Esto da inicio a una retroalimentación positiva entre los vientos alisios y la temperatura superficial del mar (TSM), de modo que el aumento de TSM debilita los vientos lo cual permite un desplazamiento de agua hacia el Este y por ende un nuevo incremento de la TSM.

Entre los posibles indicadores de El Niño, el desplazamiento de agua cálida en el Pacífico ecuatorial se identifica en este trabajo como un índice oceánico para estudiar la previsión y evolución del evento. Se considera estudiar este índice a través del volumen de agua cálida (VAC) descrito inicialmente por Meinen y McPhaden (2000).

El VAC ha sido definido como el agua de mar con temperatura mayor a $20^{\circ} \mathrm{C}$ entre latitudes $5^{\circ} \mathrm{S}-5^{\circ} \mathrm{N}$ y longitudes $120^{\circ}$ $\mathrm{E}-80^{\circ} \mathrm{W}$ (Meinen y McPhaden, 2000). Es importante notar que el desplazamiento vertical de las isotermas es fundamental en la expansión del VAC, a pesar de que sea sólo del orden de algunos metros. El VAC cubre una zona muy extensa del Pacífico ecuatorial por lo que una pequeńa variación vertical de su límite inferior (la isoterma de $20^{\circ} \mathrm{C}$ ), produciría un gran cambio del VAC.

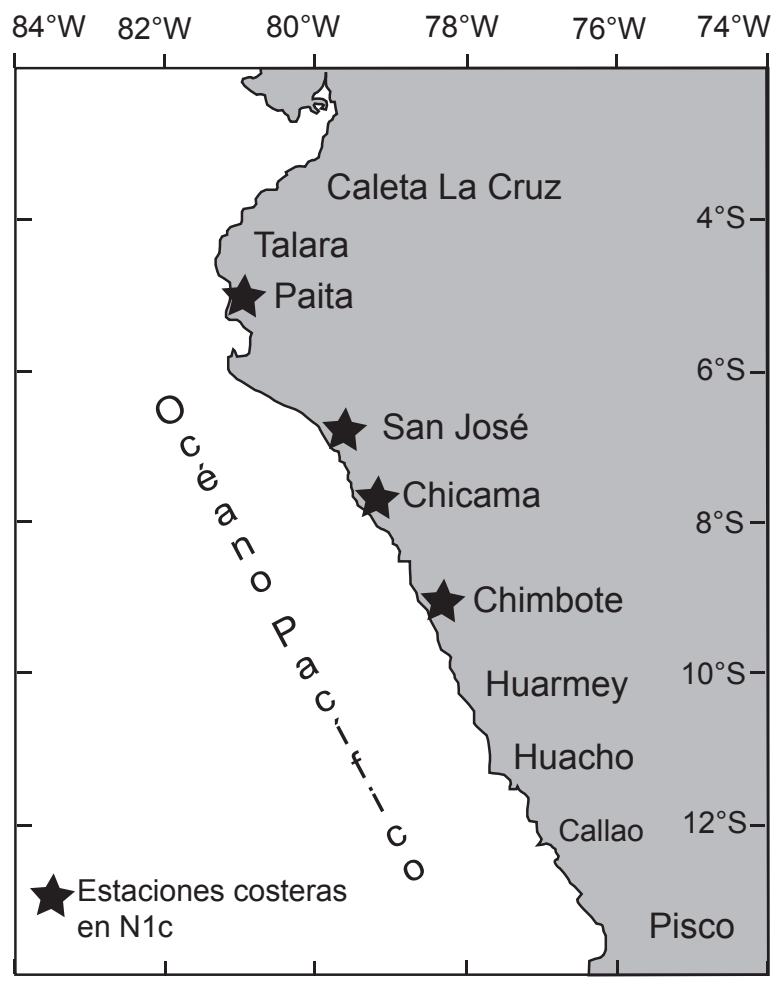

Figura 2. Ampliación de la Región Niño 1 costera y las estaciones costeras del IMARPE de donde se obtuvieron los datos de temperatura superficial del mar.
El objetivo del presente trabajo fue desarrollar un modelo empírico para la previsión de la Temperatura Superficial del Mar (TSM) en la Región Niño 1. Dicho modelo se basa en la hipótesis de que el volumen de agua cálida en el Pacífico ecuatorial está directamente relacionado a El Niño y afecta con un desfase temporal a la TSM frente al Perú.

\section{Materiales y métodos}

Se definieron dos zonas de estudio:

i) La zona del Pacífico Ecuatorial, dentro del cual está definido el VAC, se extiende entre las latitudes $5^{\circ} \mathrm{S}-5^{\circ} \mathrm{N}$ y las longitudes $120^{\circ} \mathrm{E}-80^{\circ} \mathrm{W}$ (Fig. 1).

ii) La zona original de la Región Niño 1 se extiende entre las latitudes $10^{\circ} \mathrm{S}-5^{\circ} \mathrm{S}$ y las longitudes $90^{\circ} \mathrm{W}-80^{\circ} \mathrm{W}$. El modelo utiliza los datos registrados por las estaciones costeras de IMARPE (Paita $05^{\circ} 04^{\prime}$ 'S, San José 0646' S, Chicama $07^{\circ} 41^{\prime} S$, Chimbote $09^{\circ} 04^{\prime} S$ ). Por tal motivo los resultados del modelo son más representativos de la franja costera de la Región Niño 1, que en este trabajo fue denominada Región Niño 1 costera (Fig. 2).

Se utilizaron las siguientes variables:

i) Profundidad de la isoterma de $20^{\circ} \mathrm{C}(\mathrm{Z} 20$, en m). Esta variable se obtuvo de las boyas del proyecto conjunto Tropical Atmosphere Ocean project (TAO) / Triangle Trans-Ocean buoy Network (TRITON). Dicho proyecto es operado por la National Oceanic and Atmospheric Administration (NOAA) de Estados Unidos y Japan Marine Science and Technology Center (JAMSTEC) de Japón. La profundidad de la isoterma de $20{ }^{\circ} \mathrm{C}$ es calculada de perfiles de temperatura utilizando una interpolación lineal de profundidad versus temperatura. La distancia máxima entre temperaturas es $50 \mathrm{~m}$, siendo típicamente 20 o $25 \mathrm{~m}$ (TAO 2005).

Se trabajó con todas las boyas del proyecto TAO / TRITON entre $05^{\circ} \mathrm{S}$ y $05^{\circ} \mathrm{N}$, entre enero 1980 y octubre 2003, a intervalos diarios. La información de cada boya fue organizada en archivos separados donde se procedió a calcular promedios mensuales, promedios patrones y anomalías mensuales.

ii) Volumen de agua cálida (VAC, en $10^{14} \mathrm{~m}^{3}$ ). El VAC se define como el agua sobre la isoterma de $20^{\circ} \mathrm{C}$, por ubicarse en el centro de la termoclina ecuatorial que divide la capa cálida superficial, del agua fría subsuperficial (Kessler 1990 en Meinen y McPhaden 2000). Su extensión horizontal y vertical se haya a partir de la Z20. Los datos de la serie VAC observada de enero 1980 a mayo 2003 fueron proporcionados por M. J. McPhaden (com. per. 2002).

iii) Temperatura superficial del mar de la Región Niño 1 costera (TN1c, en $\left.{ }^{\circ} \mathrm{C}\right)$. Se promediaron las temperaturas de 4 estaciones costeras (Paita $05^{\circ} 04^{\prime}$ S, San José 06 $46^{\prime}$ S, Chicama $07^{\circ} 41^{\prime} \mathrm{S}$, Chimbote $09^{\circ} 04^{\prime} \mathrm{S}$ ) proporcionadas por la Dirección de Oceanografía Física del IMARPE. En el campo, la TSM se registra 3 veces por día en el muelle de cada estación costera. Se trabajó con datos mensuales desde enero 1980 hasta octubre 2003, empleando una regresión lineal con la estación mejor correlacionada para completar los datos faltantes de cada serie. Los datos de la estación de San José tuvieron que ser completados hacia atrás debido a que las observaciones recién iniciaron en enero 1991. El 
método de interpolación empleado fue la descomposición y desestacionalización de las series, seguidas de una regresión lineal entre las estaciones mejor correlacionadas.

\section{Análisis de datos}

El desarrollo del modelo se realizó siguiendo los procedimientos similares a los usados por Meinen y McPhaden (2000). Se estimó una relación entre el VAC y la Z20, para lo cual es importante examinar el rol de la isoterma de $20^{\circ} \mathrm{C}$ en distintos puntos del océano Pacífico ecuatorial, con el fin de hallar su relación exacta con la variación del VAC. Con este fin se calcularon las correlaciones cruzadas a diferentes rezagos entre el VAC y la serie de Z20 para todas las boyas TAO ubicadas en el Ecuador (latitud $0^{\circ}$ ), registrándose la mayor correlación de cada longitud.

Luego se estimó una relación entre la anomalía del VAC (AVAC) y la anomalía de la temperatura en la Región N1c (ATN1c). Las previsiones de la ATN1c hasta julio de 2004 fueron clasificadas en 5 condiciones mensuales con rangos equitativos (fría, neutra, cálida débil, cálida moderada y cálida fuerte). La base de datos y los algoritmos del modelo se implementaron usando la hoja de cálculo Excel.

\section{Resultados}

Inicialmente se buscó simular el VAC a partir de los datos de Z20 de una boya o combinación de boyas en el Pacífico ecuatorial. Teniendo en mente que el VAC es dependiente de Z20 se buscó además un retraso en la respuesta del VAC, de tal manera poder predecir a corto plazo la variable. Se realizaron correlaciones cruzadas entre el VAC y la serie de Z20 de todas las boyas en latitud $0^{\circ} \mathrm{N}$. El coeficiente de determinación $\left(\mathrm{r}^{2}\right)$ para cada boya se muestra en la Tabla 1.

$\mathrm{La} \mathrm{Z} 20$ en las boyas de $180^{\circ} \mathrm{W}, 170^{\circ} \mathrm{W}$, y $155^{\circ} \mathrm{W}$, es decir aquellas ubicadas en el Pacífico central, son las que muestran los mas altos $r^{2}$ con el VAC, lo que indica que las oscilaciones de Z20 en los extremos del Pacífico probablemente se deban a procesos locales de corta duración que no influyen el VAC.

Se buscó mejorar el $\mathrm{r}^{2}$ utilizando combinaciones de 2 o 3 boyas en una misma zona incluyendo las boyas de latitudes 2 a $5^{\circ}$ norte y sur. Zonalmente Z20 en el Pacífico central mostró el más alto $r^{2}$ con el VAC, mientras que meridionalmente fue en

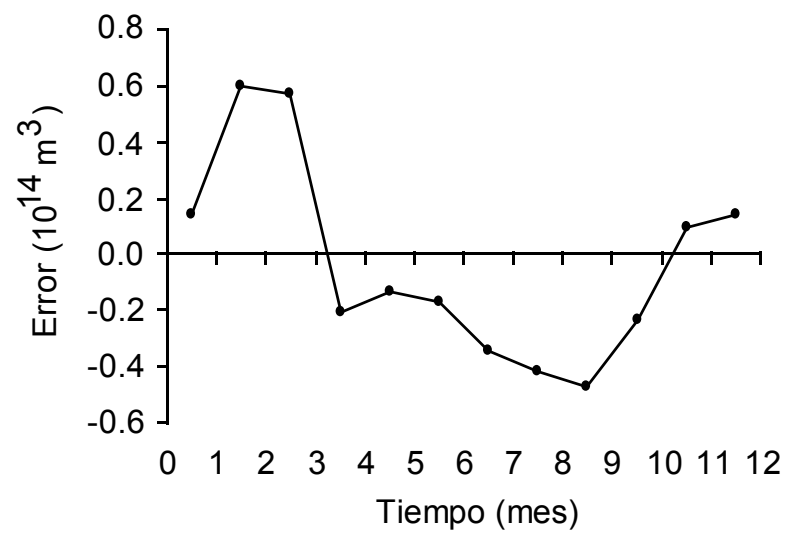

Figura 3. Promedio mensual del error del volumen de agua calida (VAC) simulado con el modelo inicial.

latitud cero. El mayor $\mathrm{r}^{2}$ con el VAC lo mostró el promedio de $\mathrm{Z} 20$ registrado en las boyas de latitud $0^{\circ} \mathrm{N}$ y longitud $180^{\circ} \mathrm{W}$, $170^{\circ} \mathrm{W}$ y $155^{\circ} \mathrm{W}$, que con $\mathrm{Z} 20$ rezagado 2 meses presentó un $\mathrm{r}^{2}$ de 0,7714 .

El modelo inicial que se utilizó con la combinación de boyas $0^{\circ} \mathrm{N}$ y $180-170-155^{\circ} \mathrm{W}$ fue:

$$
y_{\mathrm{t}}=0,0642 x_{\mathrm{t}-2}+17,661
$$

donde: $y_{\mathrm{t}}$ es el VAC, $x$ es la $\mathrm{Z} 20$ en $0^{\circ} \mathrm{N}$ y $180-170-155^{\circ} \mathrm{W}$

Se empleó este modelo para obtener una serie del VAC simulado y se comparó con el VAC observado. Los promedios mensuales de los errores mostraron una marcada estacionalidad, siendo positivos en los meses de primavera y verano austral (noviembre a marzo) (Fig. 3).

Esto implica que existe algún factor con comportamiento estacional que afecta la relación entre la Z20 en el Pacífico central y el VAC por lo cual se decidió separar la serie en dos partes: la primera parte abarcó los meses de noviembre a marzo y la segunda parte de abril a octubre. El procedimiento fue repetido y se obtuvo un $r^{2}$ de 0,7881 para noviembre-marzo y un $r^{2}$ de 0,8324 para abril-octubre. El VAC que se simuló empleando estos dos modelos tuvo un mejor ajuste con el VAC observado.

Tabla 1. Coeficientes de determinación $\left(\mathrm{r}^{2}\right)$ a diferentes rezagos entre el volumen de agua calida (VAC) y la de la isoterma de $20^{\circ} \mathrm{C}(\mathrm{Z20}$, en $\mathrm{m})$ en boyas ubicadas a $0^{\circ} \mathrm{N}$. En negrita, el mayor valor de cada longitud.

\begin{tabular}{rrrrrrrrrrr}
\hline Rezago & $147^{\circ} \mathrm{E}$ & $156^{\circ} \mathrm{E}$ & $165^{\circ} \mathrm{E}$ & $180^{\circ}$ & $170^{\circ} \mathrm{W}$ & $155^{\circ} \mathrm{W}$ & $140^{\circ} \mathrm{W}$ & $125^{\circ} \mathrm{W}$ & $110^{\circ} \mathrm{W}$ & $95^{\circ} \mathrm{W}$ \\
\hline 0 & 0,04 & 0,06 & 0,12 & 0,35 & 0,54 & $\mathbf{0 , 7 0}$ & $\mathbf{0 , 5 5}$ & $\mathbf{0 , 5 7}$ & $\mathbf{0 , 3 0}$ & 0,04 \\
1 & 0,09 & 0,14 & 0,20 & 0,52 & 0,68 & $\mathbf{0 , 7 2}$ & 0,48 & 0,52 & 0,15 & 0,00 \\
2 & 0,16 & 0,23 & 0,30 & 0,62 & $\mathbf{0 , 7 1}$ & 0,62 & 0,37 & 0,41 & 0,04 & 0,02 \\
3 & 0,23 & 0,31 & 0,38 & $\mathbf{0 , 6 4}$ & 0,66 & 0,48 & 0,26 & 0,27 & 0,00 & 0,07 \\
4 & 0,27 & 0,36 & 0,43 & 0,64 & 0,60 & 0,35 & 0,17 & 0,15 & 0,01 & 0,14 \\
5 & 0,28 & 0,38 & 0,45 & 0,62 & 0,49 & 0,24 & 0,10 & 0,06 & 0,05 & 0,21 \\
6 & 0,29 & $\mathbf{0 , 3 8}$ & $\mathbf{0 , 4 5}$ & 0,59 & 0,40 & 0,14 & 0,06 & 0,02 & 0,10 & 0,28 \\
7 & 0,32 & 0,38 & 0,44 & 0,51 & 0,30 & 0,07 & 0,03 & 0,00 & 0,15 & 0,36 \\
8 & 0,35 & 0,38 & 0,44 & 0,43 & 0,21 & 0,03 & 0,01 & 0,00 & 0,19 & 0,44 \\
9 & 0,40 & 0,38 & 0,45 & 0,33 & 0,14 & 0,00 & 0,00 & 0,01 & 0,23 & 0,51 \\
10 & $\mathbf{0 , 4 2}$ & 0,35 & 0,43 & 0,25 & 0,09 & 0,00 & 0,00 & 0,03 & 0,28 & $\mathbf{0 , 5 5}$ \\
\hline
\end{tabular}




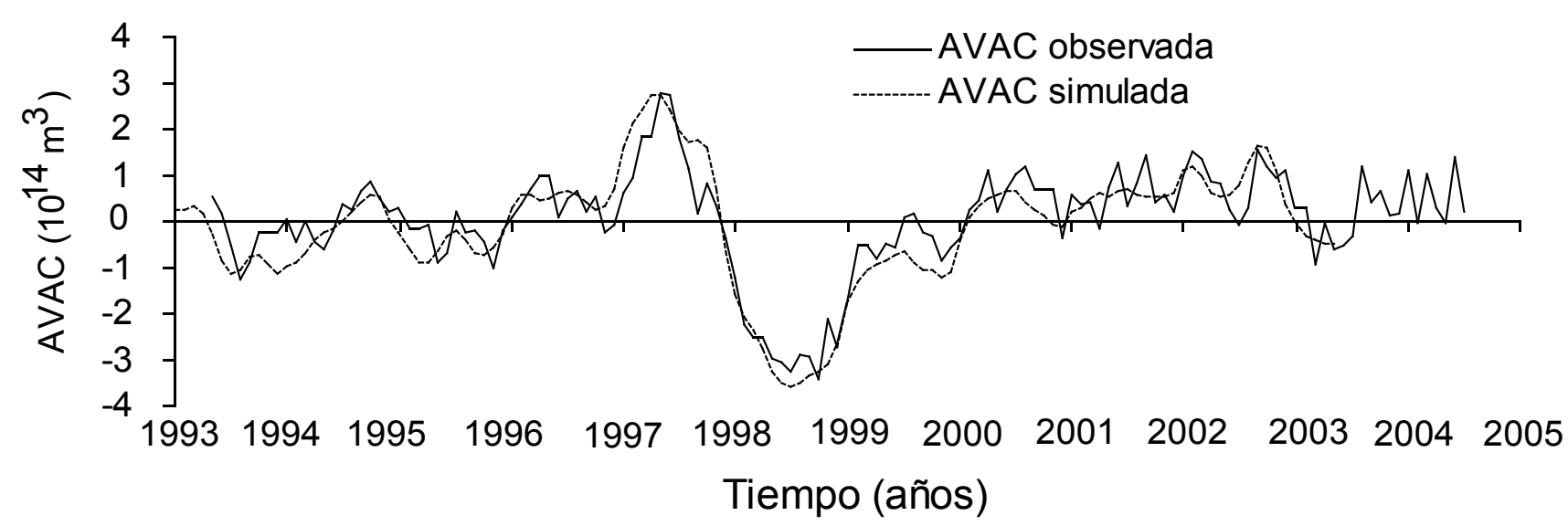

Figura 4. Variación temporal de la anomalía de volumen de agua calida (AVAC) observada y la AVAC simulada.

Para minimizar el error en la simulación del VAC, se sustrajo el promedio mensual del error respecto a la serie original. Esto disminuyó el valor máximo, mínimo y el promedio del error, mejorando el ajuste con el VAC observado. Los modelos que se aplicaron finalmente para la estimación del VAC fueron:

Para noviembre - marzo

$$
y_{\mathrm{t}}=0,0606 x_{\mathrm{t}-2}+17,787-\overline{\mathrm{e}}_{\mathrm{m}}
$$

Para abril - octubre

$$
y_{\mathrm{t}}=0,0719 x_{\mathrm{t}-2}+16,817-\overline{\mathrm{e}}_{\mathrm{m}}
$$

Donde: $y_{\mathrm{t}}$ es el VAC, $x$ es la Z20 en $0^{\circ} \mathrm{N}$ y $180-170-155^{\circ}$ $\mathrm{W}, \overline{\mathrm{e}}_{\mathrm{m}}$ es el error promedio mensual según el mes que se estima.

Luego se simuló el AVAC mostrando ser muy similar a la serie original (Fig. 4), particularmente durante El Niño de 1997-98. Sin embargo la serie simulada presentó un pequeño ruido debido a que estuvo en función a la Z20 en tres boyas, las cuales probablemente presentaron fluctuaciones asociadas a procesos locales.

El comportamiento de la AVAC fue prácticamente inverso a la Z20 en las boyas $180-170-155^{\circ} \mathrm{W}$ (Fig. 5), tendiendo a incrementar cuando la Z20 se profundiza. Esta variable alcanzó su mayor profundidad al comienzo de El Niño 1997-98 que fue cuando ocurrió la máxima AVAC simulada. Luego, durante el mismo evento se registró la mayor elevación de Z20 desde que se iniciaron las observaciones, lo cual fue acompañado por el mayor descenso de la AVAC simulada. En cuanto a los últimos meses de 2003, la AVAC simulada ha mostrado poca variación, producto de la estabilidad de la Z20.

Para ampliar el horizonte de previsión de la AVAC por 2 meses adicionales se experimentó emplear el modelo con la combinación de boyas ubicadas en latitud $2^{\circ} \mathrm{N}$ y longitud $165^{\circ} \mathrm{E}$, $180^{\circ} \mathrm{W}, 170^{\circ} \mathrm{W}$ que presentó un $\mathrm{r}^{2}$ de 0,678 con $\mathrm{Z} 20$ rezagado 4 meses. En general el modelo reprodujo bien la serie original de la AVAC aunque durante algunos periodos cortos lo sobrestimó demasiado, lo cual podría aumentar el error en la previsión de variables dependientes de la AVAC, tal como la ATN1c.

El siguiente paso fue encontrar una relación entre la AVAC y la ATN1c. Se realizó una correlación cruzada entre ambas variables que mejoró al utilizar una media móvil de 5 meses para la AVAC. Los coeficientes de determinación fueron bajos por lo que se separó los datos de la AVAC en valores positivos y negativos. Se repitió el procedimiento y los $\mathrm{r}^{2}$ incrementaron. La relación con la ATN1c fue mayor para los valores positivos de la AVAC (Fig. 6). Una relación similar fue mostrada por Meinen y McPhaden (2000) pero con la anomalía de la temperatura de la Región Niño 3.

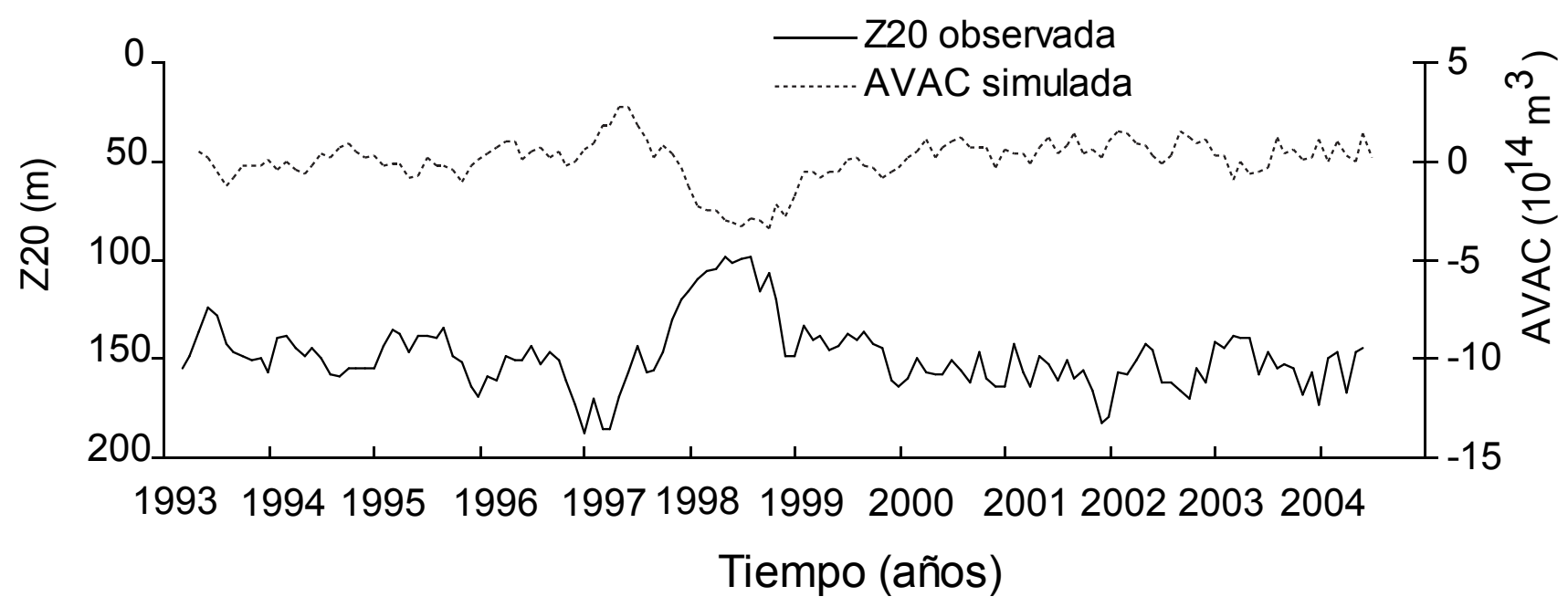

Figura 5. Variación temporal de la profundidad de la isoterma de $20{ }^{\circ} \mathrm{C}\left(\mathrm{Z} 20\right.$, en m) observada en $180-170-155{ }^{\circ} \mathrm{W}$ y la anomalía de volumen de agua calida (AVAC) simulada. 
Tabla 2. Validación del modelo empírico durante los principales eventos cálidos.

\begin{tabular}{cccccc}
\hline Período del & Mes de inicio de \\
evento cálido & $\mathrm{d}($ ATN1c) $/ \mathrm{dt}>0$ & $\begin{array}{c}\text { Error temporal en el } \\
\text { inicio de } \\
\mathrm{d}(\text { ATN1c) } / \mathrm{dt}>0\end{array}$ & $\begin{array}{c}\text { Error temporal en el } \\
\text { máximo }\end{array}$ & $\begin{array}{c}\text { Error en intensi- } \\
\text { dad del máximo }\end{array}$ \\
\cline { 5 - 6 } & Oct & 3 meses tarde & 1 mes antes & $-4,79$ & 54,68 \\
$1982-83$ & Nov & 3 meses tarde & 1 mes antes & $-1,25$ & 40,45 \\
$1986-87$ & Nov & 0 meses & 1 mes antes & $-1,41$ & 35,70 \\
$1991-92$ & Abr & No previsto & & & \\
1993 & Jun & No previsto & & \\
1994 & Mar & 3 meses tarde & 1 mes antes & $-0,71$ & 9,16 \\
$1997-98$ & Set & 0 meses & 3 meses antes & $-0,25$ & 10,12 \\
$2002-03$ & & & & & \\
\hline
\end{tabular}

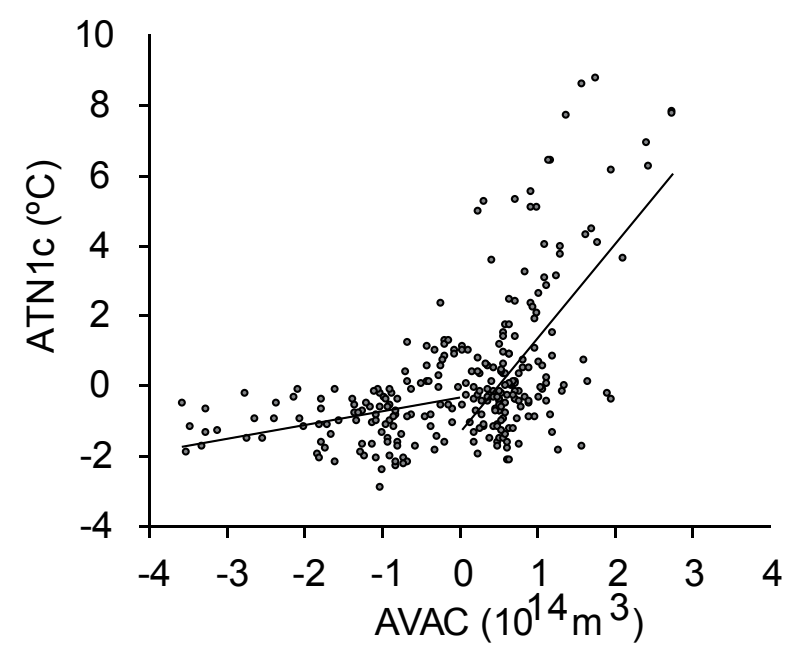

Figura 6. Relación entre la anomalía de la temperatura superficial del mar de la Región Niño 1 costera (ATN1c, en ${ }^{\circ} \mathrm{C}$ ) y la media móvil de 5 meses de la anomalía de volumen de agua calida (AVAC) rezagada 7 meses.

La AVAC fue rezagada 7 meses respecto a la ATN1c con lo cual se obtuvo el siguiente modelo:

Para una AVAC positiva $\mathrm{a}_{\mathrm{t}}=3,1696 \mathrm{~b}_{\mathrm{t}-7}-1,5808$

Para una AVAC negativa $\mathrm{a}_{\mathrm{t}}=0,4122 \mathrm{~b}_{\mathrm{t}-7}-0,2991$

Donde: a es la ATN1c, b es la AVAC

Aplicando este modelo se simuló la ATN1c desde 1980 hasta 2003 (Fig. 7).
Para validar el modelo, se analizaron los principales eventos cálidos (Tabla 2), encontrándose que el modelo reproduce satisfactoriamente la mayoría de eventos El Niño. La Tabla 2 muestra que el modelo simuló aceptablemente las fechas de inicio y máximo de los eventos cálidos previstos con un error máximo de 3 meses, pero subestimó la intensidad de los máximos con un rango de error entre $0,25^{\circ} \mathrm{C}$ y $4,79^{\circ} \mathrm{C}$.

El Nińo 1997-98 fue bien reproducido salvo un ligero retardo en el inicio del evento. El caso de El Niño 1982-83 fue muy distinto, lográndose simular el momento de inicio pero no la intensidad del evento. Esto sugiere que el proceso de estos dos fenómenos El Nińo fue distinto: en el caso del evento 1982-83 la recarga y descarga del VAC fue mucho menor que en 199798, sugiriendo que el VAC podría tomar distintas formas físicas al proyectarse hacia la costa de Sudamérica, resultando en que aguas cálidas lleguen a la costa en 1982—83, pero en menor cantidad. La retroalimentación de estas aguas cálidas en 1982—83 con la atmósfera posiblemente fue mayor debido a la época del año en que ocurrió, lo cual contribuyó a incrementar la temperatura más de lo esperado. Cabe notar que en 1982 la ATN1c empezó a tender hacia valores positivos en octubre (mes de primavera en la Región N1c) mientras que en 1997 fue en marzo (mes de otoño en la Región N1c), es decir en épocas opuestas del año.

Históricamente la primera mitad de la década del 90 fue como ninguna otra, debido al suceso de tres consecutivos eventos $\mathrm{El}$ Niño, lo cual es aun inexplicable para muchos científicos, que postulan que es necesario un periodo de recarga de calor antes de un nuevo evento. Esta falta de recarga de calor resultó que el

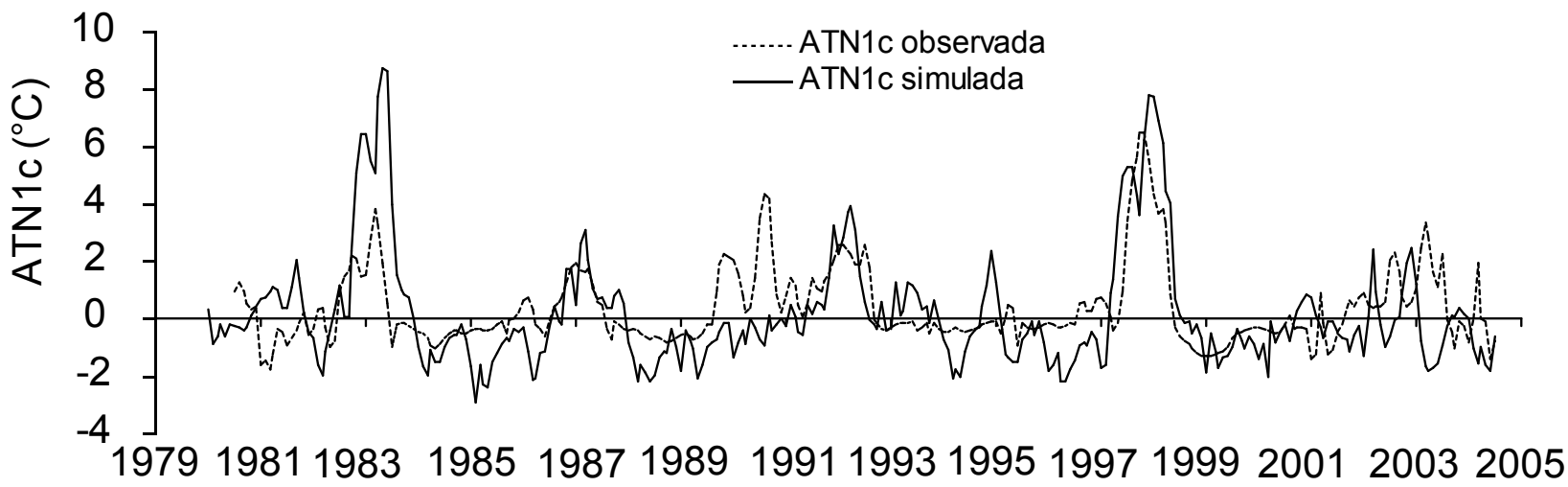

\section{Tiempo (años)}

Figura 7. Comparación entre la anomalía de la temperatura superficial del mar de la Región Niño 1 costera (ATN1c, en ${ }^{\circ} \mathrm{C}$ ) simulada a partir del la anomalía de volumen de agua calida (AVAC) observado, y la ATN1c observada. 


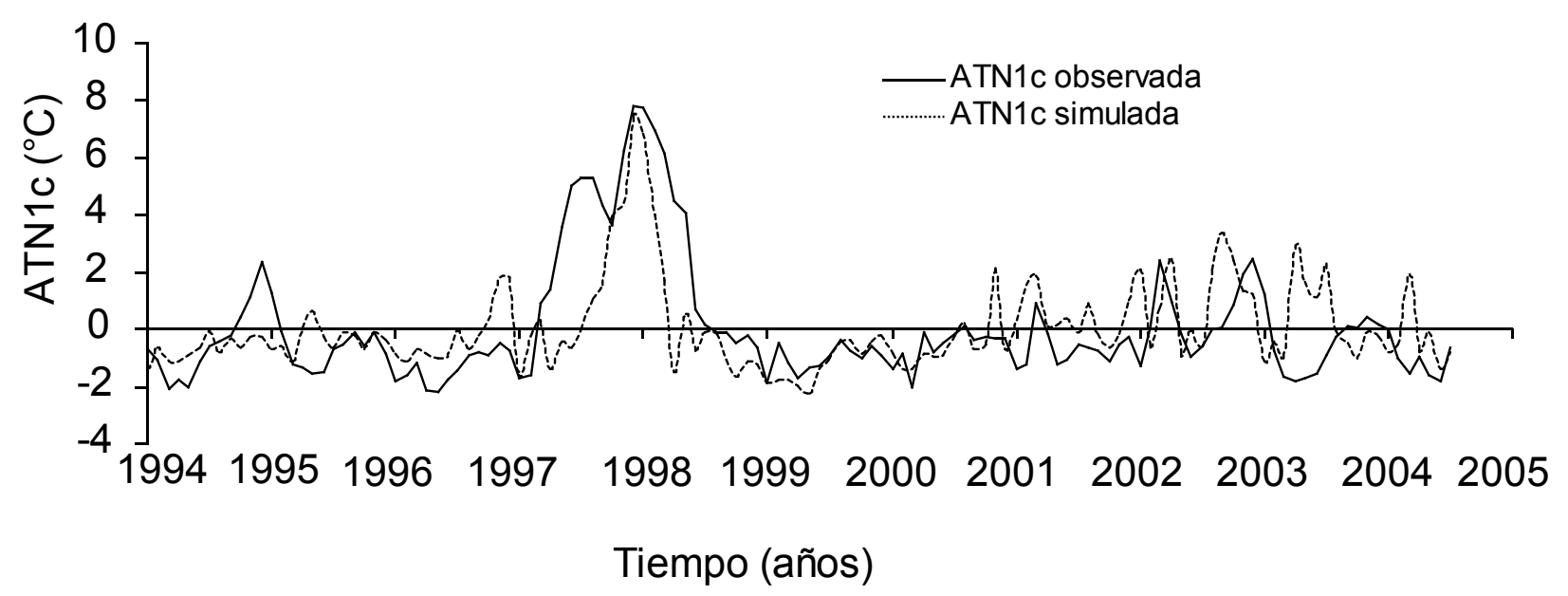

Figura 8. Comparación entre la anomalía de la temperatura superficial del mar de la Región Niño 1 costera (ATN1c, en ${ }^{\circ} \mathrm{C}$ ) simulada a partir de la anomalía de volumen de agua calida (AVAC) simulada, y la ATN1c observada.

modelo no reprodujera los El Niño 1993 y 1994 . De manera contraria, el modelo simuló calentamientos que nunca ocurrieron en 1990 y 1991 basado en dos periodos de elevados valores positivos de la AVAC. Esto implica que la expansión del VAC no se manifestó en la Región N1c y por lo tanto hubo una mayor expansión vertical y/o meridional del VAC en alguna otra región del Pacífico ecuatorial. De cualquier modo, el periodo entre 1990 y 1995 requiere de un mayor estudio y quizás la postulación de nuevas hipótesis sobre El Niño.

Utilizando los desfases entre la Z20 y la AVAC, y entre la AVAC y la ATN1c, fue posible prever la ATN1c hasta con 9 meses de anticipación (Fig. 8). El modelo estimó bien las tendencias a mediano plazo, observándose desde 1999 la gradual transición de valores negativos de la ATN1c hacia valores ligeramente positivos. Sin embargo, debido a que está previsión de la ATN1c utilizó una AVAC simulada, el error aumentó en las estimaciones mensuales. El calentamiento asociado a El Nińo 1997-98 fue simulado por un periodo más corto de lo que ocurrió, debido a que el VAC simulado fue ligeramente menor al observado.

La causalidad entre las series de la ATN1c y de la AVAC, la cual a su vez es explicada por la serie de la Z20 en 180 - 170_ $155^{\circ} \mathrm{W}$, se muestra claramente en la figura 9. Por ejemplo el máximo de la ATN1c prevista para el verano 2004 es producto del aumento de AVAC en agosto de 2003, el cual es el resultado de la profundización de la Z20 en junio 2003. En cuanto a la estimación de recientes eventos cálidos, el modelo reprodujo el breve aumento de la ATN1c que ocurrió en marzo y abril 2002, así como El Niño 2002-03 que se manifestó en la costa de Perú por un periodo corto.

Sin embargo se estimó un nuevo aumento anómalo de la TN1c para abril—julio 2003 (Fig. 10) que nunca ocurrió a pesar de que la AVAC simulada no tuvo errores significativos. La explicación probablemente se deba a la participación de otras variables que intervengan en el proceso de desplazamiento de agua cálida (vientos, flujos oceánicos, gradiente térmico, etc.). Como ya se mencionó, a una escala mensual este modelo empírico no simula con precisión la variación de la ATN1c, pero sigue relativamente bien las tendencias a mediano plazo. Los efectos de El Nińo 2002-03 sobre la costa peruana duraron sólo 3 meses y la previsión para el periodo entre noviembre 2003 a julio 2004 mostró una TN1c fluctuando cerca de su promedio patrón (Tabla 3). La tabla 3 muestra que el modelo simuló condiciones frías desde agosto 2003 hasta julio 2004, a excepción de noviembre-diciembre 2003 (condición neutra) y marzo 2004 (condición cálida).

\section{Discusión}

El modelo empírico basado en el VAC es una herramienta útil para la previsión de tendencias de la ATN1c dado que está

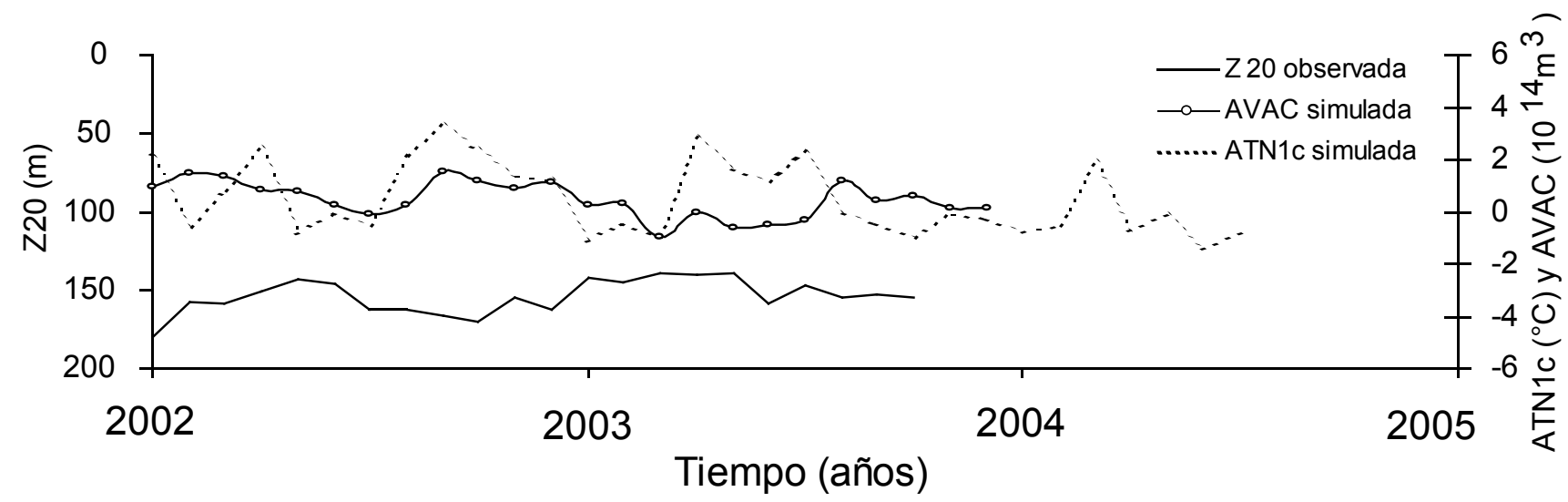

Figura 9. Variación temporal de la profundidad de la isoterma de $20^{\circ} \mathrm{C}(\mathrm{Z} 20$, en $\mathrm{m})$ observada, la AVAC simulada y la previsión de la ATN1c simulada hasta julio 2004. 


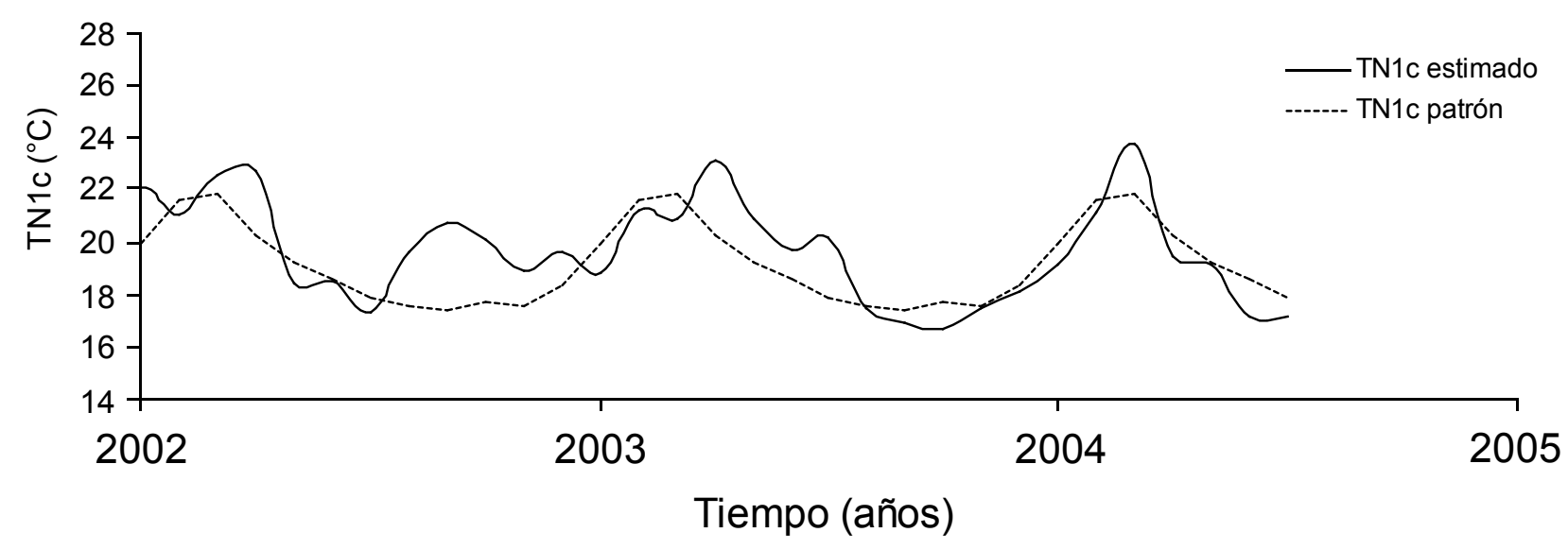

Figura 10. Variación temporal de la temperatura superficial del mar de la Región Niño 1 costera (ATN1c, en ${ }^{\circ} \mathrm{C}$ ) promedio patrón y la previsión de la TN1c simulada basada en la AVAC simulada hasta julio 2004.

basado en una variable cuyos cambios responden al estado general del Pacífico tropical y subtropical. Como es de esperar, a corto plazo las previsiones muestran mayores errores, especialmente cuando la AVAC es negativa. Sin embargo, el modelo reprodujo bien el momento en que la ATN1c empezó a ascender antes de El Nińo y también los momentos en que ocurrieron los picos de los eventos. En este sentido, el modelo empírico es más confiable a mediano plazo en comparación con los modelos autoregresivos, los cuales son más confiables a corto plazo. La intensidad de los eventos también fue bien reproducida con excepción de los casos 1982 - 83, 1993, y 1994 donde posiblemente el proceso dominante no fue la recarga y descarga del VAC. En este caso no se sostendría la hipótesis de Cane y Zebiak (1985) y Wyrtki (1986) quienes propusieron que hay una recarga de agua cálida antes de un El Niño y que después del evento el contenido de calor es bajo.

Existen diversas maneras de mejorar el modelo empírico. Durante la fase experimental del trabajo se realizaron distintas pruebas con las variables, incluyendo probar la relación entre la AVAC y la ATN1c durante verano e invierno austral, también se probó estimar datos para la región Niño $1+2$ pero el modelo funcionó mejor para la Región N1c y sin hacer distinciones para el verano e invierno. Una prueba interesante sería trabajar con una

Tabla 3. Previsiones de la temperatura superficial del mar de la Región Niño 1 costera (ATN1C, en ${ }^{\circ} \mathrm{C}$ ) simulada basada en la anomalía de volumen de agua calida (AVAC) simulada.

\begin{tabular}{ccc}
\hline Mes / Año & ATN1c $\left({ }^{\circ} \mathrm{C}\right)$ & Condición mensual \\
\hline Ago 2003 & $-0,40$ & fría \\
Set 2003 & $-0,60$ & fría \\
Oct 2003 & $-0,99$ & fría \\
Nov 2003 & 0,16 & neutra \\
Dic 2003 & 0,01 & neutra \\
Ene 2004 & $-0,66$ & fría \\
Feb 2004 & $-0,24$ & fría \\
Mar 2004 & 1,78 & cálida débil \\
Abr 2004 & $-0,89$ & fría \\
May 2004 & $-0,24$ & fría \\
Jun 2004 & $-1,50$ & fría \\
Jul 2004 & $-0,66$ & fría \\
\hline
\end{tabular}

zona reducida del VAC para que abarque un área más restringida a la línea ecuatorial y al continente sudamericano. También se podría incorporar datos de la Z20 entre 1980 y 1993 para definir la relación entre dicha variable y el VAC en ese periodo con el fin de variar el modelo según las décadas.

Para agregar nuevas variables al modelo se requeriría de un estudio previo de relaciones causa-efecto de El Niño donde se buscaría identificar los procesos de mayor importancia, además del traslado de agua cálida hacia el Pacífico Este.

La interacción océano-atmósfera juega un rol importante dando lugar a una retroalimentación entre los dos procesos, que pueden acelerar y/o prolongar un calentamiento. Es posible que el modelo empírico haya estimado tarde El Niño 1997-98 debido a que no tomó en consideración los efectos aceleradores de la atmósfera. Además el evento estimado fue más corto de lo observado posiblemente porque en la realidad el debilitamiento de los vientos contribuye a mantener las aguas cálidas por mayor tiempo. De antemano sería necesario hacer un análisis de los vientos en la Región N1c o en una zona cercana para estudiar el efecto de esta variable sobre la expansión del VAC hacia el este.

Algunos calentamientos cortos observados en la Región N1c podrían ser generados por ondas Kelvin que no afectan significativamente al VAC, por lo que no serían previstos por el modelo. Por otro lado, las ondas Kelvin demoran 2 a 3 meses en cruzar el océano Pacífico, y si es que la Z20 se profundizara, su efecto sobre la ATN1c simulada por el modelo se manifestaría 9 meses después.

Durante la fase experimental se trabajó con datos de la región Niño $1+2$ para cubrir una zona donde no actúan procesos costeros, pero la relación entre la ATSM y la AVAC fue menor que cuando se usaron datos de la región N1c. Esto sugiere que aguas cálidas desplazadas hacia el Este tienen mayor influencia en las zonas más costeras, mientras lejos de la costa posiblemente haya mayor influencia de grandes corrientes $u$ otros procesos de macroescala.

En suma podemos decir que:

1) El volumen de agua cálida (VAC) está mejor relacionado con la profundidad de la isoterma de $20^{\circ} \mathrm{C}$ (Z20) en el Pacífico central, y la correlación disminuye conforme los datos de la Z20 provengan de los extremos del Pacífico ecuatorial. 
2) La relación entre la Z20 y el VAC mejora al separar los datos de la Z20 en dos partes: noviembre a marzo, y abril a octubre. Probablemente esto se deba a que los flujos meridionales estacionales de entrada al VAC tengan una mayor extensión espacial que los flujos de salida.

3) La relación entre la anomalía del volumen de agua cálida (AVAC) y la anomalía de temperatura en la Región N1c (ATN1c) es mayor si se emplea un modelo para los valores positivos de la AVAC y otro modelo para los valores negativos.

4) La variación del VAC influye sobre la ATN1c varios meses después, mostrando una mayor correlación con un desfase de 7 meses.

5) La previsión de El Niño 1997-98 tuvo una duración más corta que lo observado, posiblemente por procesos de mantenimiento de calor en la Región N1c aún cuando el VAC inició su proceso de descarga. La relación entre las variables AVAC, Z20 y ATN1c es netamente oceánica, por lo que el modelo no considera los efectos atmosféricos, que en el caso de El Niño 1997-98 actuaron como un acelerador de los procesos de calentamiento en el océano, y mantuvieron el calor en la Región N1c mientras el VAC disminuía en el Pacífico Oeste.

6) El modelo reprodujo la intensidad de El Nińo 1997-98 el cual se inició en enero, pero no la intensidad de El Niño 1982-83 que se inició en marzo, lo cual sugiere que podría existir algún mecanismo que amplifica el efecto del VAC sobre la ATN1c durante ciertas épocas del año.

\section{Agradecimientos}

Se agradece al Dr. Michael J. McPhaden por proporcionar la serie de anomalías del VAC y por sus comentarios al respecto. De igual manera se agradece a la Dirección de Investigaciones Oceanográficas por proporcionar los datos de los laboratorios costeros de IMARPE. Finalmente se reconoce el apoyo del proyecto CENSOR y del CIMOBP, en especial de la Dra. Sara Purca y del Tco. Augusto Ingunza.

\section{Literatura citada}

Arntz, W. E. \& E. Fahrbach. 1996. El Niño. Experimento climático de la naturaleza. $311 \mathrm{p}$.

Bjerknes, J. 1969. Atmospheric teleconnections from the equatorial Pacific. Mon. Wea. Rev. 97:163-172.

Cane, M. A. \& S. E. Zebiak. 1985. A theory for El Niño and the Southern Oscillation. Science. 228(4703):1085-1087.

McPhaden, M. J. 2003. Tropical Pacific Ocean heat content variations and ENSO persistence barriers. Geophysical Research Letters. 30(9):1480, doi:10.1029/2003GL016872.

Meinen, C.S. \& M.J. McPhaden. 2000. Observations of warm water volume changes in the equatorial Pacific and their relationship to El Nino and La Nina. J. Climate. 13:3551-3559.

TAO (Tropical Atmosphere Ocean Project). 2005. (en línea). Data Display. NOAA. Pacific Marine Environmental Laboratory. $<$ http://www.pmel.noaa.gov/tao/data deliv/ > . Acceso al 03/11/05.

Wyrtki, K. 1986. Water displacements in the Pacific and genesis of El Niño cycles. J. Geophys. Res. 91:7129-7132. 